\title{
Radial viscous fingering in miscible Hele-Shaw flows: A numerical study
}

\author{
Ching-Yao Chen, ${ }^{1, *}$ C.-W. Huang, ${ }^{2}$ Hermes Gadêlha, ${ }^{3}$ and José A. Miranda ${ }^{3, \dagger}$ \\ ${ }^{1}$ Department of Mechanical Engineering, National Chiao Tung University, Hsinchu, Taiwan, Republic of China \\ ${ }^{2}$ Department of Mechanical Engineering, National Yunlin University of Science and Technology, Yunlin, Taiwan, Republic of China \\ ${ }^{3}$ Departamento de Física, LFTC, Universidade Federal de Pernambuco, Recife, Pernambuco 50670-901 Brazil
}

(Received 15 February 2008; revised manuscript received 24 April 2008; published 17 July 2008)

\begin{abstract}
A modified version of the usual viscous fingering problem in a radial Hele-Shaw cell with immiscible fluids is studied by intensive numerical simulations. We consider the situation in which the fluids involved are miscible, so that the diffusing interface separating them can be driven unstable through the injection or suction of the inner fluid. The system is allowed to rotate in such a way that centrifugal and Coriolis forces come into play, imposing important changes on the morphology of the arising patterns. In order to bridge from miscible to immiscible pattern forming structures, we add the surface tensionlike effects due to Korteweg stresses. Our numerical experiments reveal a variety of interesting fingering behaviors, which depend on the interplay between injection (or suction), diffusive, rotational, and Korteweg stress effects. Whenever possible the features of the simulated miscible fronts are contrasted to existing experiments and other theoretical or numerical studies, usually resulting in close agreements. A number of additional complex morphologies, whose experimental realization is still not available, are predicted and discussed.
\end{abstract}

DOI: 10.1103/PhysRevE.78.016306

PACS number(s): 47.54.-r, 47.20.Ma, 47.11.-j, 64.75.-g

\section{INTRODUCTION}

Among natural nonequilibrium growth processes, the viscous fingering problem in Hele-Shaw cells has attracted much attention [1] since the seminal work by Saffman and Taylor [2]. This classic hydrodynamic instability emerges when a less viscous fluid pushes a more viscous one in the narrow gap of a Hele-Shaw cell, leading to the formation of nontrivial fingerlike structures. In the outward radial flow version of the problem the less viscous fluid is injected at the center of the cell. Experiments involving the radial flow of immiscible fluids demonstrate that as the size of the fluidfluid interface grows outward, fingers spread and start to split at their tips, creating fanlike, branched patterns [3-5]. Stateof-the-art boundary integral [6] and conformal mapping [7] numerical simulations have accurately reproduced the structures observed in such experiments.

Researchers have also studied the complementary case of the usual outward radial flow where a blob of a more viscous fluid, surrounded by a less viscous fluid, is sucked and drawn radially inward into a sink located at the center of the HeleShaw cell. Laboratory experiments of inward flow using immiscible fluids $[3,4]$ show the growth of fingering patterns which are very different from those arising in outward radial flow: multiple penetrating fingers of the less viscous fluid compete, and eventually one of them reach the center, before all the more viscous fluid is sucked out. In contrast to the outward flow case, tip splitting of inwards fingers is not detected. Boundary integral numerical simulations have also been performed for the suction of immiscible fluids in HeleShaw cells $[8,9]$. However, these numerical studies focused on the understanding of the regularizing effects of surface tension, and not exactly on the precise morphological de-

\footnotetext{
*chingyao@mail.nctu.edu.tw

†jme@df.ufpe.br
}

scription of the patterns. Equivalent experimental and numerical studies using miscible fluids are still inexistent.

Although the majority of the studies of unstable displacement in radial Hele-Shaw flows have been undertaken with immiscible fluids, some work has also been done addressing the outward flow with miscible fluids. A few experimental investigations detected the development of (i) flowerlike patterns [10] qualitatively similar to ones found in immiscible outward radial flow, and (ii) fractallike structures $[11,12]$. The miscible patterns obtained in Refs. $[11,12]$ are typically more ramified and present fingers that are thinner than their immiscible counterparts [3-5]. Another peculiar feature of these miscible structures is the observation of finger interpenetration. Other than these few morphological features, not much has been revealed experimentally. On the theoretical side, most of the work on outward radial miscible flows are restricted to purely analytical calculations addressing the linear stability of the mixing front $[10,13,14]$. The theoretical description of fully nonlinear stages of the miscible radial displacement through numerical simulations has been overlooked. In addition to providing useful insight about the morphology of possible miscible patterns, a numerical study could also offer a useful link between miscible and immiscible radial flows by the introduction of the so-called Korteweg stresses [15-20] which mimic surface tensionlike effects in miscible systems.

An alternative way of obtaining complex radial viscous fingering patterns can be provided by rotating the Hele-Shaw cell around an axis perpendicular to the plane of the flow. In this case the interface instability is driven by the density difference between the fluids. Since the original work by Schwartz [21] this problem has been largely investigated, both experimentally and theoretically (see, for instance, Refs. [22-26], and references therein). The rotating flow patterns exhibit a variety of shapes: they may change from wide teardroplike structures, to thin filamented arms presenting swelled ends, or to a branched, backbone architecture with nearly constant finger widths. The corresponding problem 
with miscible fluids has also been investigated through numerical simulations $[27,28]$. Since both the morphology of the resulting patterns and the driving forces involved are so distinct when injection, suction, and rotation act separately, one interesting possibility would be to consider the simultaneous action of injection (or, suction) plus rotation in miscible radial flows, where distinct morphologies and unexplored nonlinear behavior would certainly arise.

Still regarding the rotating Hele-Shaw situation, it is worth noting that relatively little attention has been paid to the effects due to the Coriolis force, which is usually neglected as in Refs. [22-28]. Curiously, with the exception of the work performed in [21], only very recently the role of the Coriolis force in rotating Hele-Shaw flows has been examined [29-34]. The inclusion of the Coriolis force follow two different approaches: in Refs. [21,33,34] the Coriolis force contribution is included in an ad hoc manner, so that it is added directly in a gap-averaged two-dimensional Darcy's law. On the other hand, in Refs. [29-32] a more rigorous and systematic procedure is followed, where the Coriolis force term is introduced already at the level of the threedimensional Navier-Stokes equation. Comparison between these two approaches supports the generality of the firstprinciples method employed in [29-32], indicating that the model employed in [21] can lead to inaccurate predictions already at the early linear level. A final point of interest refers to a suitable description of the advanced time stages of the interfacial evolution when the Coriolis force is significant. Existing numerical studies of both immiscible [21] and miscible $[33,34]$ flows in rotating Hele-Shaw cells including Coriolis follow Schwartz's ad hoc approach, and not the more general one proposed in Refs. [29-32]. In this sense, the fully nonlinear stages of the problem have not yet been appropriately modeled.

In this work the dynamics of the diffusing interface separating two miscible fluids in a radial Hele-Shaw cell is studied by intensive and highly accurate numerical simulations. We examine the interplay among injection, suction, diffusive, Korteweg stresses, centrifugal, and Coriolis effects in determining the shape of the fingering patterns. In particular, the role of the Coriolis force is included by following the theoretical model presented in Refs. [29-32]. The remainder of the paper is organized as follows: Section II formulates our theoretical approach, presents the governing equations, and describes the numerical scheme we have implemented to study the problem. Section III presents and discusses our numerical results: Sec. III A focus on the outward radial flow in which one of the fluids is injected into the Hele-Shaw cell, while Sec. III B analyzes the corresponding inward flow case, in which the inner fluid is sucked from the cell. Several morphological behaviors are introduced, and a number of interesting pattern forming phenomena are revealed and discussed. A brief summary of our main results and conclusions are presented in Sec. IV.

\section{BASIC EQUATIONS AND NUMERICAL APPROACH}

Consider a Hele-Shaw cell of gap thickness $b$ containing two miscible, incompressible, viscous fluids (see Fig. 1). De-
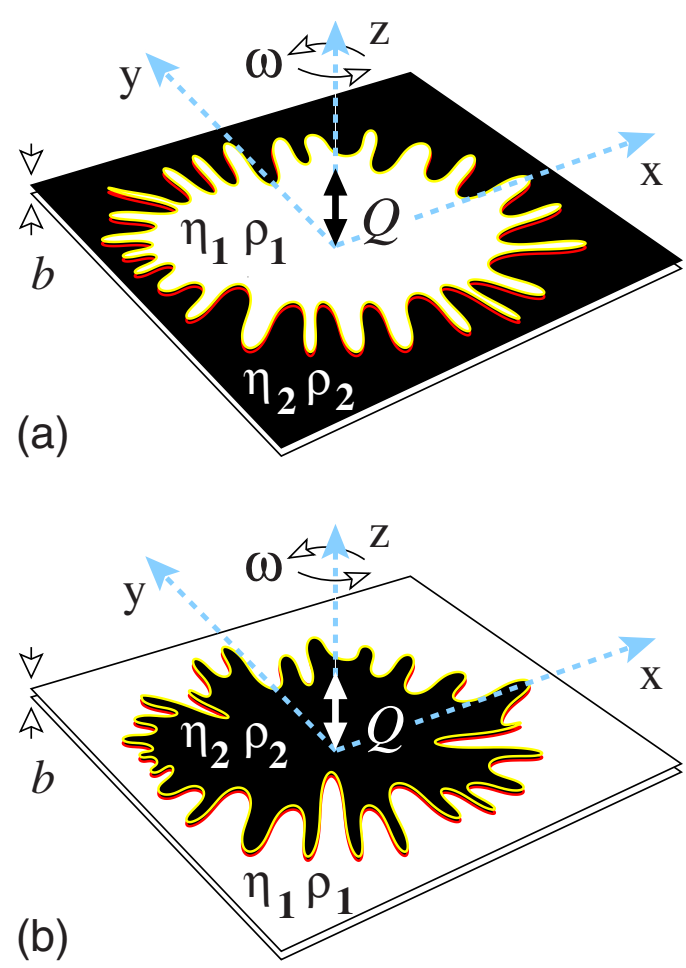

FIG. 1. (Color online) Schematic representation of radial flow in a Hele-Shaw cell with miscible fluids. The inner fluid can be injected (outward flow) or sucked (inward flow) at the origin of the coordinate system with a constant aerial rate $Q$, and the cell may rotate in the counterclockwise direction around the $z$ axis with angular velocity $\omega$. In (a) the less viscous and lower density fluid (light color) is the inner fluid, while in (b) the inner fluid (dark color) is more viscous and has higher density.

note the densities and viscosities of the fluids, respectively as $\eta_{1}, \eta_{2}$ and $\rho_{1}, \rho_{2}$, and assume that $\eta_{2}>\eta_{1}$ and $\rho_{2}>\rho_{1}$. In Fig. 1 fluid 2 is the dark fluid, while fluid 1 is the light one. Both outward and inward radial flows are considered, where injection as well as suction are performed at a constant flow rate $Q$, equal to the area covered per unit time. The lower (upper) plate is located at $z=0(z=b)$, where the $z$ axis points in the direction perpendicular to the plates. The Hele-Shaw cell can rotate in the counterclockwise direction with constant angular velocity $\boldsymbol{\omega}=\omega \hat{\mathbf{z}}$ about the $z$ axis, where $\hat{\mathbf{z}}$ is a unit vector pointing along this axis. Initially, the mixing interface is a circle, and a Cartesian coordinate system $(x, y, z)$ is defined in such a way that its origin is located at the center of this circular region. Driven by the combined action of injection (or suction) and rotation, as time progresses the fluids begin to mix diffusively giving rise to different types of complex interfacial patterns.

The equations governing the dynamical evolution of the system are the so-called gap averaged Hele-Shaw flow equations which, in a reference frame rotating with the cell, are $[17,27,28,31]$ $\boldsymbol{\nabla} \cdot \mathbf{u}=0$ 


$$
\begin{gathered}
\boldsymbol{\nabla} \mathcal{P}-\rho \omega^{2} \mathbf{r}-\frac{\mathcal{D}}{\mathcal{C}} \hat{\mathbf{z}} \times\left(\boldsymbol{\nabla} \mathcal{P}-\rho \omega^{2} \mathbf{r}\right) \\
=-\frac{12 \eta}{b^{2} \mathcal{C}} \mathbf{u}+\boldsymbol{\nabla} \cdot\left[\hat{\delta}(\boldsymbol{\nabla} c)(\nabla c)^{T}\right], \\
\frac{\partial c}{\partial t}+\mathbf{u} \cdot \nabla c=D \nabla^{2} c .
\end{gathered}
$$

Equation (1) expresses the incompressibility condition, where $\mathbf{u}(x, y)=\int_{0}^{b} \mathbf{v}(x, y, z) d z / b$ denotes the two-dimensional (2D) gap averaged velocity, and $\mathbf{v}(x, y, z)$ is the full threedimensional (3D) velocity field. An augmented Darcy's law is expressed by Eq. (2) where $\mathcal{P}$ is the total pressure including the hydrodynamic pressure $p$ and the additional pressure $\Pi$ due to Korteweg stresses, i.e., $\mathcal{P}=p+\Pi(c)$, where [17]

$$
\begin{aligned}
\Pi(c)= & \frac{\hat{\delta}}{3}\left[\left(\frac{\partial c}{\partial x}\right)^{2}+\left(\frac{\partial c}{\partial y}\right)^{2}\right]+\frac{2 \gamma}{3}\left(\frac{\partial^{2} c}{\partial x^{2}}+\frac{\partial^{2} c}{\partial y^{2}}\right) \\
& +\frac{2 \xi \eta}{3}\left[\frac{\partial}{\partial x}\left(\frac{D}{1-\xi c} \frac{\partial c}{\partial x}\right)+\frac{\partial}{\partial y}\left(\frac{D}{1-\xi c} \frac{\partial c}{\partial y}\right)\right]
\end{aligned}
$$

with $\xi=\left(\rho_{2}-\rho_{1}\right) / \rho_{2}, \hat{\delta}$ and $\gamma$ denoting the two constant Korteweg stress coefficients. The Korteweg stress contribution is given by the second term on the right-hand side of Eq. (2), and also by the additional pressure $\Pi$. Both centrifugal and Coriolis forces are taken into account on the left-hand side of Eq. (2), where $\mathbf{r}$ is the position vector of a fluid element. The gap averaged concentration equation is given by Eq. (3), where the concentration of the fluid 1 is represented by $c$, and $D$ is the constant diffusion coefficient.

The Korteweg stress contribution, which is not accounted for by the standard Navier-Stokes stress tensor of a Newtonian fluid, is related to concentration gradients that arise at the mixing region separating two miscible fluids. When a pair of miscible fluids are brought into contact, a fairly steep concentration gradient "interface" is formed between them. This interface is regarded not as a singular surface but rather as a diffuse boundary of effectively finite thickness, whose local equilibrium and transport properties, particularly the concentration, vary continuously across this interfacial transition region. This raises the possibility for the presence of something similar to an interfacial tension between miscible fluids. In Ref. [15] the history of the subject is presented over more than a century with many experimental illustrations. Such stresses were first postulated back in 1901 by Korteweg [16] who, on the basis of an ad hoc constitutive equation, suggested the existence of what can be phenomenologically described as a transient surface tension at the miscible interface. More recently, an interesting experimental work by Joseph [15] on miscible displacement renewed interest in Korteweg's original work, where it has been found that drops of water rising in glycerin are characterized by shapes that resemble those commonly seen in immiscible flows. This stimulated a host of further research in this area, with strong experimental $[19,20]$ and numerical $[28,35]$ evidence that these stresses do mimic surface-tension-like effects in miscible fluids.
The seminal work by $\mathrm{Hu}$ and Joseph [17] formulated the gap averaged equations [similar to our Eqs. (1)-(3)] to account for Korteweg stresses in miscible Hele-Shaw flows. In Ref. [17] the original momentum equation is supplemented by a nontrivial driving force involving gradients of the concentration, and by a modified pressure [a conventional one plus a "concentration pressure"] as represented in Eqs. (2) and (4). In this work, even though all cases we simulate are legitimately miscible, we use the Korteweg stress to reintroduce surface-tension-like effects into the miscible scenario. Therefore, we can simulate radial miscible displacements with different magnitudes for the Korteweg stress effect, just like people study immiscible flows with different surface tensions. This provides a very useful tool which allows us to make a parallel between our simulated miscible patterns under strong Korteweg effects and existing numerical and experimental results for radial Hele-Shaw flow with immiscible fluids.

Another noteworthy point refers to the generalized Darcylike law shown in Eq. (2). As usual, it can be obtained by averaging a 3D Navier-Stokes equation over the gap direction. As shown in Ref. [29], although the "standard" inertial terms $[\partial \mathbf{v} / \partial t+(\mathbf{v} \cdot \nabla) \mathbf{v}]$ appear in the original Navier-Stokes equation, they are later dropped by virtue of the assumed smallness of some reduced Reynolds numbers. On the other hand, the Coriolis force term survives under such gap averaged procedure. In fact, under the customary circumstances of rotating Hele-Shaw flows, the Coriolis force effects related to a rotational Reynolds number are much more relevant than other inertial contributions. Therefore, even though the "standard" inertial terms are negligible, and do not contribute to Eq. (2), Coriolis effects are indeed significant and can play an important role in rotating Hele-Shaw flows. The typical values of the rotational Reynolds numbers we use in our simulations are not too large (they vary between 0 and 0.07 ), but are consistent with the magnitudes commonly found in real experiments in rotating Hele-Shaw cells [23], and are capable of producing the characteristic type of chiral growth expected from Coriolis force effects.

The Coriolis force prefactors $\mathcal{C}$ and $\mathcal{D}$ in Eq. (2) are expressed as [31]

$$
\begin{gathered}
\mathcal{C}=\frac{\sinh \sqrt{12 \mathrm{Re}}-\sin \sqrt{12 \mathrm{Re}}}{2 \operatorname{Re} \sqrt{12 \operatorname{Re}[\cosh \sqrt{12 \mathrm{Re}}+\cos \sqrt{12 \mathrm{Re}}]},} \\
\mathcal{D}=\frac{1}{2 \operatorname{Re}}\left\{1-\frac{\sinh \sqrt{12 \mathrm{Re}}+\sin \sqrt{12 \mathrm{Re}}}{\sqrt{12 \operatorname{Re}}[\cosh \sqrt{12 \mathrm{Re}}+\cos \sqrt{12 \mathrm{Re}}]}\right\} .
\end{gathered}
$$

We point out that, in contrast to the immiscible case studied in Ref. [31], in Eqs. (5) and (6) the parameter Re defines a modified Reynolds number for the mixture, which depends on the local concentration, on the dimensionless viscosity difference between the fluids, and also on the Reynolds numbers of the fluids as 


$$
\operatorname{Re}=\frac{\rho \omega b^{2}}{12 \eta}=\left\{c\left[\frac{1+A}{1-A}\right]^{c-1} \operatorname{Re}_{1}+(1-c)\left[\frac{1+A}{1-A}\right]^{c} \operatorname{Re}_{2}\right\},
$$

where

$$
\operatorname{Re}_{1}=\frac{\rho_{1} \omega b^{2}}{12 \eta_{1}}, \quad \operatorname{Re}_{2}=\frac{\rho_{2} \omega b^{2}}{12 \eta_{2}}, \quad A=\frac{\eta_{2}-\eta_{1}}{\eta_{2}+\eta_{1}}
$$

denote the rotational Reynolds numbers of fluids 1 and 2, and the viscosity contrast, respectively. The Reynolds numbers presented in Eq. (8) work as controlling parameters that regulate the strength of the Coriolis force, which is absent when $\operatorname{Re}_{1}=\operatorname{Re}_{2}=0$. In deriving Eq. (7) we have followed the standard approach used in Refs. [36,37], and assumed that the viscosity of the mixture varies exponentially with concentration, whereas its density has a linear dependence with $c$ according to

$$
\begin{gathered}
\eta(c)=\eta_{1} \exp [(\mathcal{R}(1-c)], \\
\rho(c)=c \rho_{1}+(1-c) \rho_{2},
\end{gathered}
$$

with $\mathcal{R}=\ln \left(\eta_{2} / \eta_{1}\right)$ representing a viscosity parameter. It is worth noting that the Reynolds number of the mixture defined in Refs. $[33,34]$ does not depend on the concentration.

At this point we take a moment to discuss the validity and limitations of the Hele-Shaw model as expressed by the gap averaged Eqs. (1)-(3). A very important requirement for the validity of the theoretical approach we use refers to the fact that the velocity profile across the Hele-Shaw cell gap needs to remain parabolic $[38,39]$. In addition, it is assumed that the Taylor dispersion [40] is significant, so that the concentration gradients are not high across the gap [17,39]: since there is no net flux across the gap direction ( $z$ axis) it is reasonable to assume that the concentration is independent of z. In fact, it has been shown in Refs. [39,42-46] that, if the magnitude of the characteristic Péclet number [relative magnitude of convective and diffusive effects] of the system is not larger than a certain upper bound, the miscible HeleShaw problem is accurately described by Eqs. (1)-(3). Under such circumstances the gap averaged concentration equation (3) can be reasonably approximated by its original 3D form, where $\mathbf{v}$ is replaced by $\mathbf{u}[17,18]$. It should be pointed out that very recently a more rigorous approach for the derivation of the gap averaged concentration equation (which also results in the same form as the equivalent 3D equation) has been proposed in Ref. [41], where it has been shown that the Péclet number should be suitably large for the averaging to work.

In any case, as extensively discussed in Refs. $[28,39,42-46]$, if the Péclet number is higher than the upper bound value mentioned above, a narrow finger grows in the central region of the gap, so that the velocity profile is not simply parabolic, leading to the formation of strong concentration gradients in the $z$ direction. This defines a truly threedimensional problem, where Eqs. (1)-(3) are no longer valid. We stress that the values of the Péclet number we use in our numerical simulations are safely below the typical upper bound value for Hele-Shaw flows $\left[\leqslant O\left(10^{4}\right)\right][28]$, but still sufficiently large $\left[O\left(10^{2}\right)-O\left(10^{3}\right)\right]$ to ensure the applicability and reliability of Eqs. (1)-(3) in accurately describing our physical problem. At this Péclet number regime the good agreement between the Hele-Shaw model with the 3D Stokes model and actual experiments, as shown in Refs. [44-46], provides a solid verification for the validity of the Hele-Shaw approach as described by Eqs. (1)-(3). Additional validation of the depth-averaging approach and of our numerical methodology has been provided by the fact that highly nonlinear numerical simulations for miscible displacements in rotating [28] and lifting [35] Hele-Shaw cells compare quite well with the corresponding immiscible patterns obtained by experiments and boundary integral simulations, when sufficiently high Korteweg stresses are introduced.

In order to render the governing equations (1)-(3) dimensionless, an arbitrary length $L[47,48]$, and the density difference $\Delta \rho=\rho_{2}-\rho_{1}$ are taken as characteristic scales. In addition, we scale the viscosities and the flow velocity with $\eta_{1}$ and $Q / 2 \pi L$, respectively. Together with a characteristic pressure $6 \eta_{1} Q / \pi b^{2}$, the dimensionless set of the governing equations takes the form

$$
\begin{gathered}
\boldsymbol{\nabla} \cdot \mathbf{u}=0 \\
\boldsymbol{\nabla} \mathcal{P}=-\eta[E \mathbf{u}+F(\hat{z} \times \mathbf{u})]+\rho \Omega \mathbf{r}+\boldsymbol{\nabla} \cdot\left[\delta(\nabla c)(\nabla c)^{T}\right], \\
\frac{\partial c}{\partial t}+\mathbf{u} \cdot \nabla c=\frac{1}{\mathrm{Pe}} \nabla^{2} c
\end{gathered}
$$

where $E=\mathcal{C} /\left(\mathcal{C}^{2}+\mathcal{D}^{2}\right)$, and $F=\mathcal{D} /\left(\mathcal{C}^{2}+\mathcal{D}^{2}\right)$. Note the definition of additional dimensionless parameters, namely the Péclet number Pe, the rotation number $\Omega$, and the Korteweg constant $\delta$ which are respectively defined as

$$
\mathrm{Pe}=\frac{Q}{2 \pi D}, \quad \Omega=\frac{\pi \Delta \rho \omega^{2} b^{2} L^{2}}{6 \eta_{1} Q}, \quad \delta=\frac{\pi \hat{\delta} b^{2}}{6 \eta_{1} Q L^{2}} .
$$

Thus, the most general version of the problem requires the definition of the six dimensionless parameters listed in Eqs. (8) and (14). We point out that the values of the parameters used in our simulations are absolutely consistent with the typical order of magnitudes of the corresponding physical quantities measured in existing laboratory experiments and predicted by other theoretical studies.

For the purpose of solving the governing equations numerically, it is convenient to recast them into the well-known streamfunction-vorticity formulation $(\psi, \zeta)$ $[17,27,33,37,49]$. By employing such an approach Eqs. (12) and (13) can be rewritten as

$$
\nabla^{2} \psi=-\zeta
$$




$$
\begin{aligned}
\zeta= & -\mathcal{R} \nabla \psi \cdot \nabla c+\frac{F \mathcal{R}}{E}\left[\frac{\partial \psi}{\partial y} \frac{\partial c}{\partial x}-\frac{\partial \psi}{\partial x} \frac{\partial c}{\partial y}\right]-\frac{1}{E}\left[\left(\frac{\partial F}{\partial x}\right.\right. \\
& \left.\left.-\frac{\partial E}{\partial y}\right) \frac{\partial \psi}{\partial y}-\left(\frac{\partial E}{\partial x}+\frac{\partial F}{\partial y}\right) \frac{\partial \psi}{\partial x}\right]-\frac{\Omega}{E \eta}\left[y \frac{\partial c}{\partial x}-x \frac{\partial c}{\partial y}\right] \\
& -\frac{\delta}{E \eta}\left[\frac{\partial c}{\partial x}\left(\frac{\partial^{3} c}{\partial x^{2} \partial y}+\frac{\partial^{3} c}{\partial y^{3}}\right)-\frac{\partial c}{\partial y}\left(\frac{\partial^{3} c}{\partial x \partial y^{2}}+\frac{\partial^{3} c}{\partial x^{3}}\right)\right]
\end{aligned}
$$

where the streamfunction has been split into a rotational $(\phi)$ and a potential component $\left(\phi_{p o t}\right)$, so that $\psi=\phi+\phi_{p o t}$. If Schwartz's more informal approach is employed, it can be shown [31] that $E=1$ and $F=2 \mathrm{Re}$. Under such circumstances Eq. (16) reproduces the corresponding equations for the vorticity derived in Refs. [33,34]. Note that as a result of employing the streamfunction-vorticity formulation the additional pressure $\Pi$, and with it the second Korteweg stress constant $\gamma$ [see Eq. (4)] are eliminated, so that $\delta$ is the only additional parameter resulting from the Korteweg stress terms. The situation in which Korteweg stresses are neglected is obtained by setting $\delta=0$, while higher magnitudes of $\delta$ should result in miscible patterned structures close in appearance to their immiscible counterparts.

The rotational part of the streamfunction is smooth and can be obtained with high accuracy, while the potential part is related to a flow singularity at a source (or sink) located at the origin making accurate computations more difficult near these locations. The dimensionless potential velocity field associated to a source or sink can be formally written as

$$
u_{\text {pot }}=\frac{1}{r} .
$$

However, to avoid numerical instabilities near $r=0$ in Eq. (17) we smooth out the point source or sink by distributing their strength in a Gaussian way over a small circular core region. To accomplish this, we consider a "Gaussian source" which is characterized by a core size $\sigma=0.075$ so that the dimensionless potential radial velocity is expressed as

$$
u_{p o t}=\frac{1}{r}\left[1-\exp \left(-r^{2} / \sigma^{2}\right)\right] .
$$

For more details about the Gaussian source scheme, the reader is directed to Refs. [37,50]. Note that all the velocity distributions have the same functional form for the cases of injection (source) and suction (sink), but the velocity directions are obviously reversed.

Our numerical simulations for both injection and suction begin with an initial condition given by a stable circle of a given radius $r$ centered on the axis of rotation (origin of the $x-y$ plane). To set the initial condition we follow the approach originally proposed in Ref. [13], and use the basestate axisymmetric solution for an stable injection situation, which is represented by an error functionlike distribution [see Eq. (15) in Ref. [13]]. Within such an approach, the duration of the stable injection is given by $t=r^{2} / 2$. More specifically, for injection we start with a circular core of radius $r=r_{c}=0.1$, so that the initial condition is taken as the base state at time $t=0.005$. On the other hand, for suction we use a circle of initial radius $r=1$, so that the stable injection time to produce this initial state is $t=0.5$. In order to break the artificial symmetry of the system, we impose a small amplitude perturbation around the initial interface location. To accomplish this, a small amount of random noise is added at all grid points positioned near the 0.5 concentration location so that $c=0.5+\epsilon$ at that region, where $\epsilon$ $=0.01 \sin (2 \pi N)$ denotes the perturbation, and $N$ stands for a set of random numbers between 0 and 1 . Throughout this work, unless otherwise stated, we apply the same set of random numbers $N$ to ensure the same initial condition for the situations under study.

For the case of injection a square computational domain with width of two characteristic lengths is chosen, so that $-1 \leqslant x \leqslant+1$ and $-1 \leqslant y \leqslant+1$. To minimize artificial effects of the Gaussian source, we start the simulation with an initial radius of $r_{c}=0.1$, that is slightly larger than the core size of the Gaussian source $\sigma$. As mentioned earlier, this initial condition corresponds a stable injection time period of 0.005 . The inner fluid is injected at the origin of the coordinate system, and the simulations are terminated when the inner fluid reaches a distance of 10 grid points away from the computational boundaries, in such a way that for injection the boundary conditions are prescribed as

$$
\begin{aligned}
& x= \pm 1: \phi=0, \quad \frac{\partial c}{\partial x}=0, \\
& y= \pm 1: \phi=0, \quad \frac{\partial c}{\partial y}=0 .
\end{aligned}
$$

As discussed above, the initial condition for the case of suction (sink flow) is assumed to be a unit radius circular blob of the inner fluid which is withdrawn from the origin. To avoid effects from the computational boundaries at early time stages, we slightly extend the computational domain in such a way that for suction we consider $-4 / 3 \leqslant x \leqslant+4 / 3$ and $-4 / 3 \leqslant y \leqslant+4 / 3$. In addition, as long as the mixing interface does not reach the Gaussian core, the artificial effects of the Gaussian velocity distribution will be insignificant. In a word, the numerical treatments for the sink and source flows are identical and the boundary conditions (19) and (20) are applied, but with a slightly extended computational domain for the sink flow.

We close this section by outlining the numerical scheme we use, which is based on a combination of spectral methods and compact finite differences. Much more details about these particular numerical methods can be found in wellknown books on the subject [51,52] and also in Refs. $[50,53,54]$. The simultaneous application of these two highly accurate numerical methods is essential to reproduce the fine and intricate miscible fingering structures successfully. Regarding the particular choice of the boundary conditions [Eqs. (19) and (20)], they consider that the streamfunction is zero for all the computational boundaries. Nevertheless, to apply the spectral method along the $x$ direction, we need to modify the boundary condition as $\partial \phi / \partial x=0$. The validity of this modified condition is achieved by carefully keeping the mixing interface far away from the computational boundaries at all times, so that the vorticity and streamfunction induced 
(a)

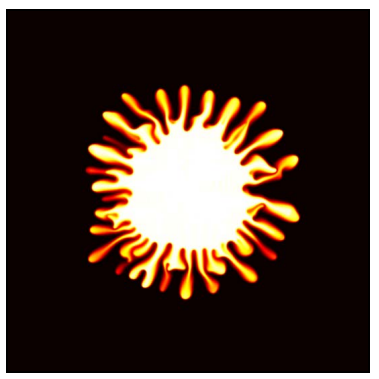

(d)

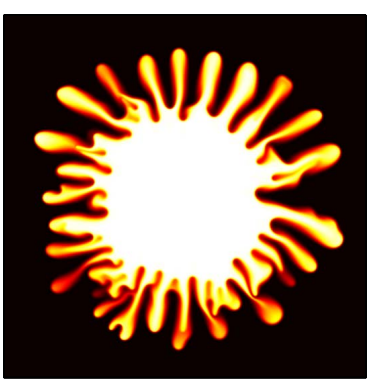

(b)

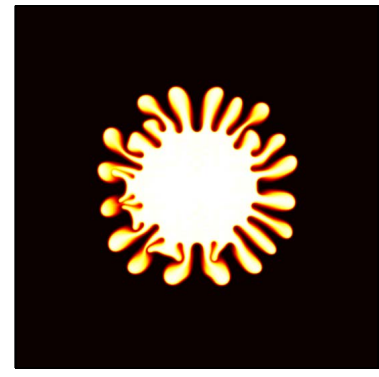

(e)

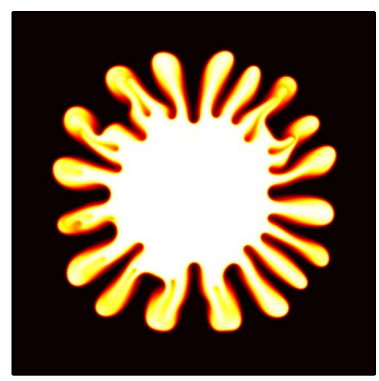

(c)

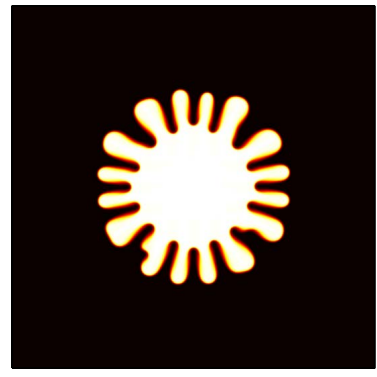

(f)

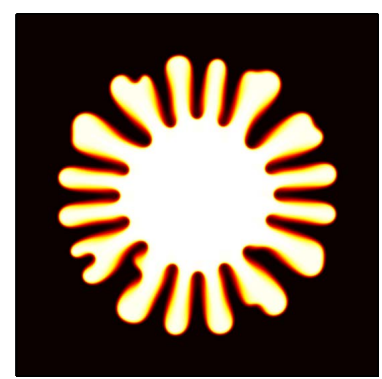

FIG. 2. (Color online) Concentration images at times $t=0.1$ (top row) and $t=0.2$ (bottom row), $A=0.874$, and $\mathrm{Pe}=10^{3}$ for $\delta=0[(\mathrm{a})$ and (d) $]$; $\delta=-10^{-5}[(\mathrm{~b})$ and (e) $] ; \delta=-2.15 \times 10^{-5}[(\mathrm{c})$ and (f) $]$.

by the concentration gradients are vanishingly small, and negligible near such boundaries. All this is confirmed by the nearly zero values of the streamfunction obtained from the actual numerical output data. The streamfunction, Eq. (15), is solved by a pseudospectral method $[51,52]$, and a Galerkintype discretization of cosine expansion of $c$, and $\phi$ are employed in the $x$ direction. In the $y$-direction discretization is accomplished by sixth-order compact finite differences [53]. The latter is also used to evaluate the vorticity equation (16). In addition, a third-order Runge-Kutta procedure [55] on time and spatial sixth order compact finite difference schemes are employed to solve the concentration equation [Eq. (13)], and advanced in time. It should be noted that, unlike many schemes based on the velocity and pressure variables, the mass conservation properties are not a concern for the present numerical approach, since the formulation of the governing equations in terms of the vorticity and streamfunction satisfies the continuity equation [Eq. (11)] identically. The numerical code has been successfully used for miscible flow in other geometries [27,37,56,57], and is quantitatively validated by comparing the growth rates with the values obtained from the linear stability theory in a plane front $[37,50,54]$. More details on the implementation and quantitative validation of these schemes are specifically provided by Refs. [18,50,54].

\section{RESULTS AND DISCUSSION}

\section{A. Outward radial flow}

We begin our analysis by focusing on the most traditional case in which the inner fluid is less viscous, and injected against the more viscous outer fluid, so that the viscosity contrast $A=0.874$, and $\mathrm{Pe}=10^{3}$. This particular value of the viscosity contrast results in significant fingering and it is actually the largest value of $A$ we can simulate for outward radial flow; so, it will be used throughout Sec. III A. All the patterns depicted in this section are plotted inside a square frame that has the same dimensions of the entire computational domain $(-1 \leqslant x \leqslant+1$ and $-1 \leqslant y \leqslant+1)$.

First, we consider the situation in which the Hele-Shaw cell is not rotating, so that centrifugal and Coriolis effects are absent $\left(\Omega=0, \operatorname{Re}_{1}=0\right.$, and $\left.\operatorname{Re}_{2}=0\right)$. As mentioned in Sec. I the equivalent immiscible situation resulted in fanlike patterns in which wide outward fingers are formed, and tend to split at their tips. We investigate how the shape of the emerging patterns behave under miscible circumstances, and try to verify explicitly if the miscible patterns present a better resemblance to their immiscible counterparts as the Korteweg stress is added and its strength is gradually increased.

Figure 2 illustrates the effects of increasingly larger Korteweg stress parameter $\delta$ on the evolution of the miscible patterns under injection. The columns in Fig. 2 are arranged according to the value of the Korteweg stress parameter: first column [Figs. 2(a) and 2(d)] for $\delta=0$; second [Figs. 2(b) and 2(e)] for $\delta=-10^{-5}$; and third [Figs. 2(c) and 2(f)] for $\delta=-2.15 \times 10^{-5}$. The top row 2(a) -2 (c) depicts the patterns at time $t=0.1$, while the bottom row 2(d)-2(f) shows the structures formed for a later time $t=0.2$. When $\delta=0$ miscible viscous fingering patterns arise as the mixing front evolves, unfolding a process of mutual entrainment characterized by finger competition, and finger merging [see Figs. 2(a) and 2(d)]. The resulting pattern is intricate and reveals the formation of relatively thin fingering structures, which tend to become a little wider as time progresses. As detected by experiments in miscible flows driven by injection $[11,12]$, finger interpenetration is observed as shorter fingers merge to larger ones, sometimes leading to the entrapment of small 
(a)

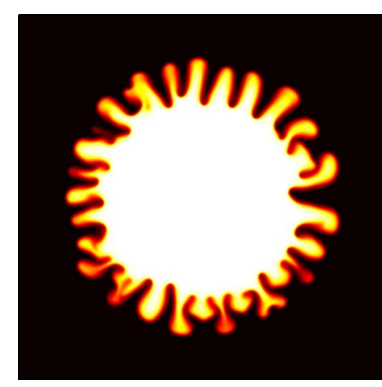

(d)

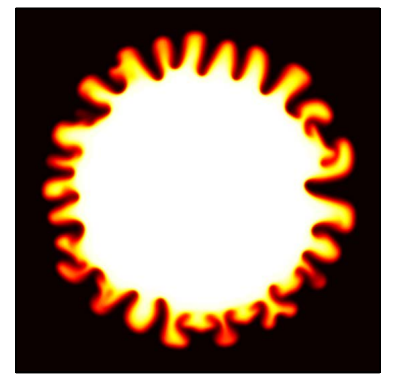

(b)

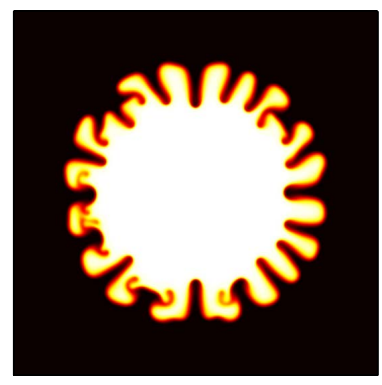

$(e)$

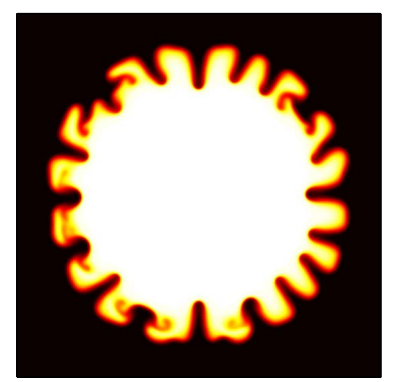

(c)

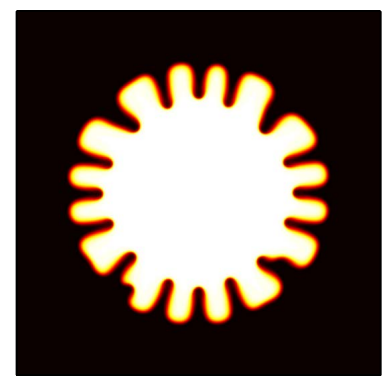

(f)

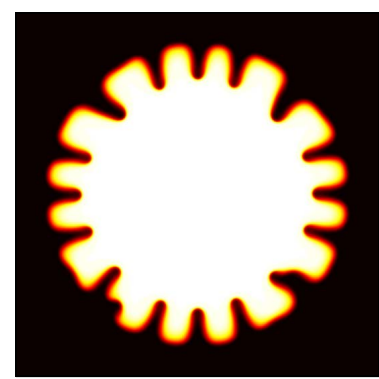

FIG. 3. (Color online) Concentration images at times $t=0.20$ (top row) and $t=0.275$ (bottom row), $A=0.874, \mathrm{Pe}=10^{3}, \Omega=20$, and $\operatorname{Re}_{1}=\operatorname{Re}_{2}=0$ for $\delta=0\left[(\mathrm{a})\right.$ and (d)]; $\delta=-10^{-5}[(\mathrm{~b})$ and (e) $] ; \delta=-2.15 \times 10^{-5}$ [(c) and (f)]. (a) $-(\mathrm{c})$ should be contrasted to Figs. 2(d) $-2(\mathrm{f})$ which have been simulated for exactly the same physical parameters and time, but for $\Omega=0$.

portions of the dark fluid inside the light one [Figs. 2(a) and 2(d) and Figs. 2(b) and 2(e)].

As $\delta$ is increased [Figs. 2(b) and 2(e) and Figs. 2(c) and 2(f)] the stabilizing role of the Korteweg stresses is disclosed, and the typical number of fingers is decreased. A more relevant effect of $\delta$ refers to its influence on the shape of the fingers: nonzero $\delta$ makes the outward fingers become wider, assuming more rounded fingertips. Since Korteweg stresses restrict the growth of the finger lengths, and also reduce their number, in order to maintain the amount of fluid flux unchanged one would expect the fingers to become wider. However, finger interpenetration can still be observed in Figs. 2(b) and 2(e) for intermediate values of $\delta$. For the case of stronger Korteweg stresses simulated in Figs. 2(c) and 2(f) the number of fingers is further reduced, and the resulting fingering patterns do resemble the ones obtained by experiments $[3,4,11]$ and numerical simulations $[6,7]$ of the analogous immiscible situation. A fanlike pattern, presenting broad fingers and showing finger tip splitting is clearly seen in Fig. 2(f). Moreover, finger interpenetration is no longer present. These results reinforce the claim that Korteweg stresses do act similar to an effective surface tension for miscible flows [19,20,56,57], by stabilizing shortest wavelengths. The shape similarity between our simulated miscible patterns shown in Figs. 2(c) and 2(f) (obtained for high Korteweg stress and largest possible viscosity contrast $A$ $=0.874$ ) and well-documented experimental results for immiscible radial flows (performed for $A=1$ in Refs. [3,4,11]) also provides an indirect validation of our theoretical approach and numerical methodology.

We proceed by analyzing the case in which in addition to injection, we consider the effects of Hele-Shaw cell rotation. Specifically, we consider that the rotational dimensionless parameter $\Omega=20$. For now we focus on centrifugally driven effects and analyze a rotational case in which the Coriolis force is not taken into account so that $\operatorname{Re}_{1}=\mathrm{Re}_{2}=0$. Figure 3 illustrates the diffusing patterns at $t=0.2$ (top row) and at $t$ $=0.275$ (bottom row) for three different values of $\delta$ : $0[$ (a) and $(\mathrm{d})] ;-10^{-5}[(\mathrm{~b})$ and $(\mathrm{e})]$; and $-2.15 \times 10^{-5}[(\mathrm{c})$ and $(\mathrm{f})]$. These are exactly the same values of $\delta$ used in the simulations shown in Fig. 2. Note that Figs. 3(a)-3(c) can be readily compared to Figs. 2(d)-2(f) also taken at $t=0.2$ and for which rotational effects were not included. By contrasting these figures we notice that the most noteworthy effect of the centrifugal force refers to its influence on the typical length of the fingers: with the inclusion of rotation, which is a stabilizing factor in this outward radial flow situation, the outward fingers become significantly less elongated. In addition, both finger interpenetration and finger tip- splitting tend to be suppressed in Fig. 3. As a result the typical fanlike pattern [Fig. 2(f)] is replaced by a fairly regular array of fingers, which are flat at their tips, resulting in a pattern that looks similar to a cog wheel [Fig. 3(c)]. Although the time taken in Figs. 3(d)-3(f) is a little longer than the time chosen in Figs. 3(a)-3(c), the shape of the patterns remain basically unchanged. The fact that we can go further in time in Fig. 3 as compared to the time considered in Fig. 2 reinforces the fact that the centrifugal force acts to stabilize the system. Even though this stabilizing effect is not really surprising, it is interesting to see how the typical fingering patterns induced by injection [Fig. 2] can have their morphology modified via the action of centrifugal effects at fully nonlinear stages. These numerical findings are in line with the analytical results of Ref. [24] where the stabilizing role of rotation is used to suppress finite time cusp singularities in the absence of surface tension for the outward radial flow of immiscible fluids. 
(a)

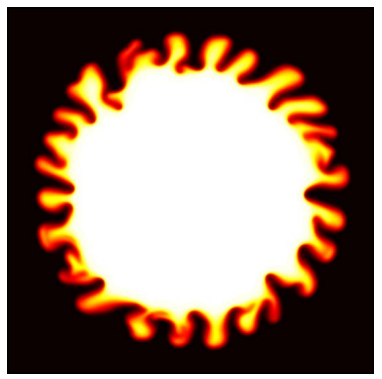

(b)

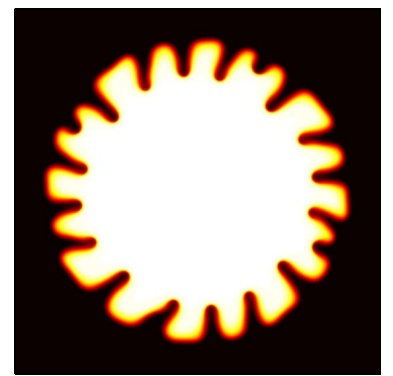

(c)

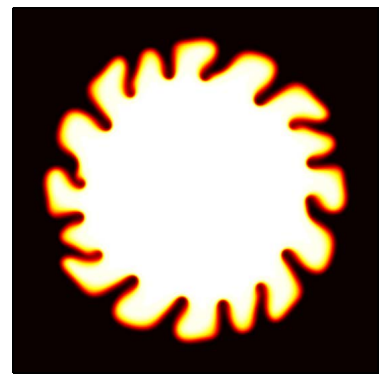

FIG. 4. (Color online) Concentration images at time $t=0.275, A=0.874, \mathrm{Pe}=10^{3}, \Omega=20$, and $\operatorname{Re}_{1}=0.01$ for (a) $\delta=0, \operatorname{Re}_{2}=0.03$; (b) $\delta$ $=-2.15 \times 10^{-5}, \operatorname{Re}_{2}=0.03$; (c) $\delta=-2.15 \times 10^{-5}, \operatorname{Re}_{2}=0.06$. (a) should be compared to Fig. 3(d), while (b) and (c) should be compared to Fig. $3(\mathrm{f})$.

We turn to the situation in which Coriolis effects are included, meaning that the Reynolds numbers of the fluids are nonzero. Figure 4 depicts the patterns obtained at time $t=0.275$, considering that $\Omega=20$ and $\operatorname{Re}_{1}=0.01$ for the cases: (a) $\delta=0, \mathrm{Re}_{2}=0.03$; (b) $\delta=-2.15 \times 10^{-5}, \mathrm{Re}_{2}=0.03$; (c) $\delta=-2.15 \times 10^{-5}, \mathrm{Re}_{2}=0.06$. Notice that the time taken in Fig. 4 is equal to the one considered in Figs. 3(d)-3(f). So, we can make an interesting parallel between these figures. By inspecting Fig. 4(a) for which $\delta=0$ we see that the outward moving fingers no longer evolve radially as in Fig. 3(d), but present an evident tendency to turn in the clockwise direction. This is a clear indication of the action of the Coriolis force: the actual spin direction of the Hele-Shaw cell is counterclockwise, thus the finger tip motion is retrograde, so that the outgrowing fingers move "backward." This is also true when we have nonzero Korteweg stresses as shown in Figs. 4(b) and 4(c) where the fingers bent toward the clockwise direction. These last two figures should be contrasted with Fig. 3(f) obtained for the same value of $\delta$, where Coriolis effects are absent. Note that the finger bending phenomenon is even more visible in Fig. 4(c) leading to a pattern that is similar to a pinwheel in appearance. This is due to the fact that a larger Reynolds number has been used for the more viscous fluid $\left(\operatorname{Re}_{2}=0.06\right)$ in Fig. 4(c). When the inner fluid is the lighter one, the Coriolis force acts to destabilize the interface by deviating the fingering structures along the azimuthal direction.
An alternative and more quantitative account of the role played by Korteweg stresses, centrifugal, and Coriolis effects in determining the behavior of the evolving mixing interface is offered by the growth of a characteristic quantity related to the perimeter of the mixing boundary region. Unlike the situations involving immiscible fluids, the mixing region between two miscible fluids is not a well-defined sharp interface, but rather a diffuse layer. Nevertheless, in the region of significant concentration gradient, the normalized mixing interfacial length can be well represented as $[27,37,56]$

$$
L_{n}(t)=\frac{1}{L_{B}(t)} \int_{S} \sqrt{\left(\frac{\partial c}{\partial x}\right)^{2}+\left(\frac{\partial c}{\partial y}\right)^{2}} d x d y,
$$

where $S$ is the entire computational domain. This quantity expresses the ratio of the length of the diffuse interface to the perimeter of the initial circular pattern (base state) $L_{B}(t)$ $=2 \pi \sqrt{2(t+0.005)}$ at a given time $t$.

Figure 5 depicts the time evolution of the normalized mixing interfacial length $L_{n}$ for $A=0.874$ and $\mathrm{Pe}=10^{3}$, for some of the situations illustrated in Figs. 2-4, (a) in the absence of rotation and for increasing values of the Korteweg stress parameter $\delta$ [related to Fig. 2]; (b) in the absence of Coriolis effects, for $\delta=-2.15 \times 10^{-5}$, and four increasing values of the dimensionless rotation number $\Omega$ [related to Figs. 2(c), 2(f), 3(c), and 3(f)]; (c) when Coriolis effects are taken into account, for $\Omega=20, \delta=-2.15 \times 10^{-5}$, (a)

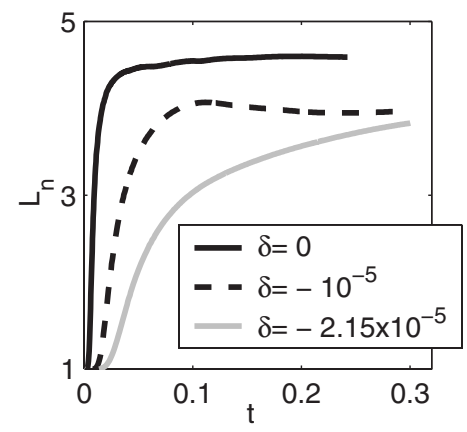

(b)

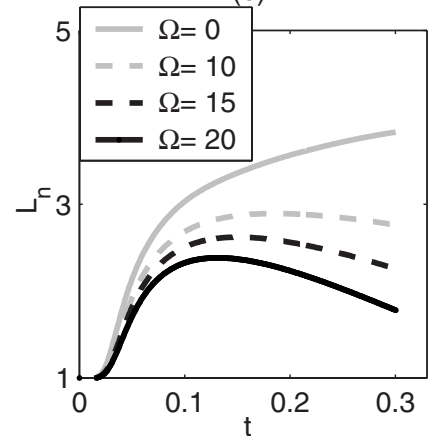

(c)

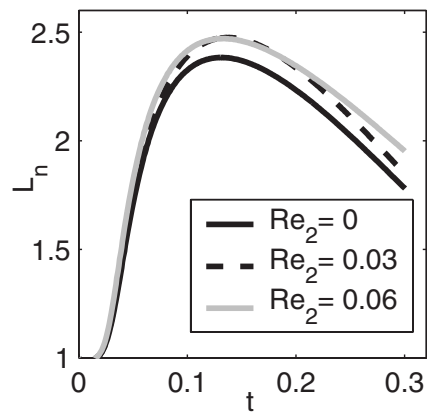

FIG. 5. Time evolution of the normalized interfacial length $L_{n}$ for $A=0.874, \mathrm{Pe}=10^{3}$, and (a) $\Omega=0, \operatorname{Re}_{1}=\operatorname{Re}_{2}=0$, and different values of $\delta$; (b) $\operatorname{Re}_{1}=\operatorname{Re}_{2}=0, \delta=-2.15 \times 10^{-5}$, and different values of $\Omega$; (c) $\Omega=20, \delta=-2.15 \times 10^{-5}$, and different values of $\operatorname{Re}_{2}$. In (c) note that if $\operatorname{Re}_{2}=0\left(\operatorname{Re}_{2}>0\right)$ then $\operatorname{Re}_{1}=0\left(\operatorname{Re}_{1}=0.01\right)$. 
(a)

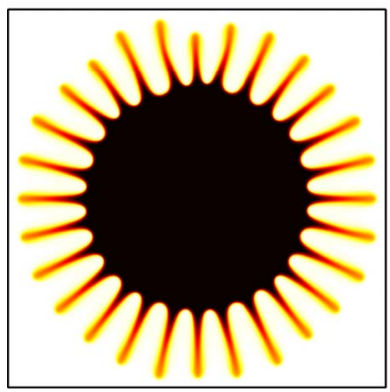

(c)

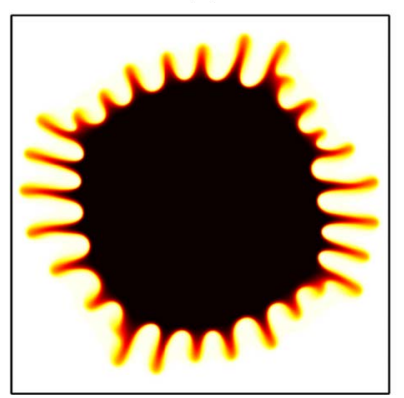

(d)

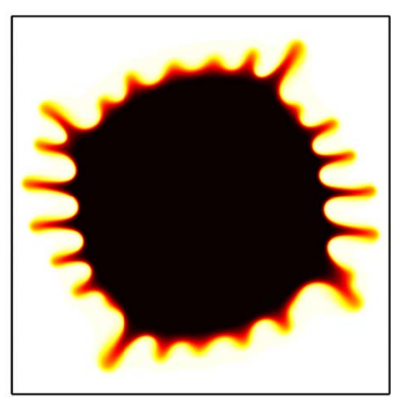

FIG. 6. (Color online) Concentration images for $A=0.874, \mathrm{Pe}$ $=2.0 \times 10^{2}$, and $\Omega=10^{2}$, when $[(a)$ and (b)] Coriolis effects are neglected $\left(\operatorname{Re}_{1}=0, \mathrm{Re}_{2}=0\right)$ for (a) $\delta=0, t=0.22$, (b) $\delta=-10^{-4}, t$ $=0.25$; and when $[(\mathrm{c})$ and (d)] the Coriolis force is taken into account $\left(\operatorname{Re}_{1}=0.01, \operatorname{Re}_{2}=0.07\right)$ considering that (c) $\delta=0, t=0.26$; (d) $\delta=-10^{-4}, t=0.27$.

and three different values of $\operatorname{Re}_{2}$ [related to Figs. 3(c), 3(f), 4(b), and 4(c)]. In Fig. 5(c) note that if $\operatorname{Re}_{2}=0\left(\operatorname{Re}_{2}>0\right)$ then $\operatorname{Re}_{1}=0\left(\operatorname{Re}_{1}=0.01\right)$.

The growth of normalized interfacial length serves as a good indicator for the intensity of fingering at the mixing interface. From Fig. 5(a) it is evident that the presence of increasingly larger Korteweg stresses tend to stabilize the diffuse interface. When $\delta=0$ we observe a very steep growth of $L_{n}$ at earlier times, followed by its "saturation" for longer times. For short times, we notice that the slope of the curves are reduced for larger $\delta$. In addition, for all times the curves for lower $\delta$ are always above the ones of higher $\delta$. Again, this is in accordance with the surface-tension-like role of the Korteweg stress, which tends to stabilize the mixing interface. It is interesting to note that for $\delta=-10^{-5}$, after an intense initial growth, $L_{n}$ reaches a maximum, and then starts a slow decrease. This behavior is due to the finger merging process as depicted in Figs. 2(b) and 2(e). On the other hand, for $\delta=-2.15 \times 10^{-5} L_{n}$ tends to increase and show no signs of saturation. This happens because finger merging and finger interpenetration are suppressed for higher $\delta$, while finger tip- splitting emerges, favoring interfacial growth [see Figs. 2(c) and 2(f)].

As to the role of rotation, since for these injection cases the inner fluid has a lower density, Fig. 5(b) reproduces the expected behavior: larger values of $\Omega$ lead to increasingly stable interfaces, resulting in lower values of $L_{n}$. Of course, these results are not surprising, but offer a more quantitative verification of the equivalent visual information provided by (a)

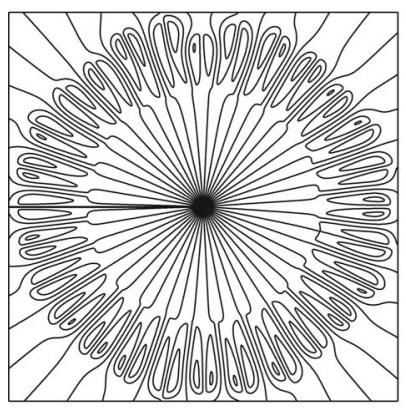

(c)

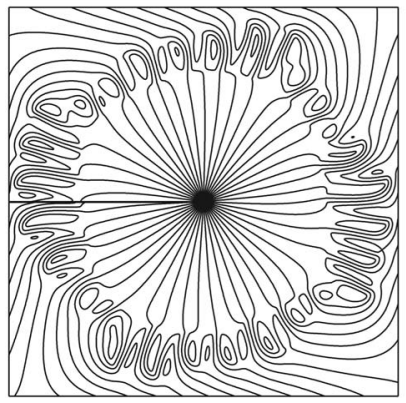

(b)

(d)

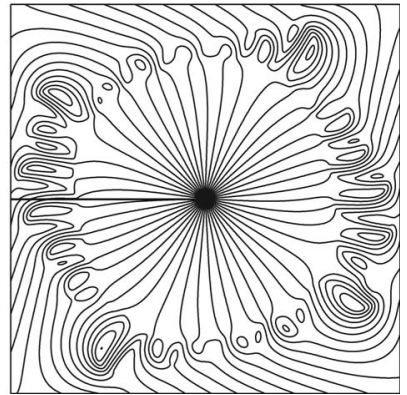

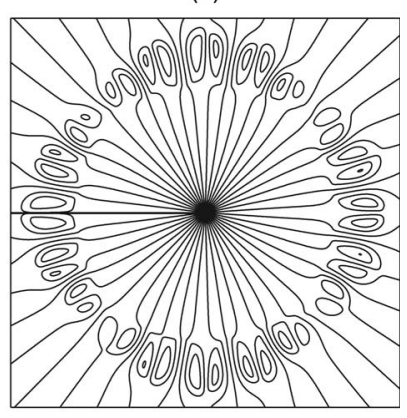

FIG. 7. Streamlines of the situations shown in Fig. 6.

the patterns shown in Figs. 2(c), 2(f), 3(c), and 3(f). However, the situation described in Fig. 5(c) regarding the influence of the Coriolis force is certainly more interesting. It is well known from previous analytical [31] and numerical [33] studies that the Coriolis force acts to stabilize the interface if the inner fluid is more dense (see also Fig. 6). On the other hand, by inspecting Fig. 5(c), it is clearly verified that, if the inner fluid is the less dense, now the Coriolis effects tend to destabilize the interface. These effects are easily captured from Fig. 5(c) where the curve for a given $\operatorname{Re}_{2}>0$ is located above the curve for $\operatorname{Re}_{2}=0$. The relatively modest destabilizing effect exhibited in Fig. 5(c) can also be visualized by contrasting Fig. 3(f) with Figs. 4(b) and 4(c). Finally, notice that the higher $\mathrm{Re}_{2}$ is the larger $L_{n}$ is, reinforcing the claim that stronger Coriolis effects lead to increased interface destabilization if the inner fluid is the lower density one.

We close this set of numerical experiments on outward radial flow by analyzing one last situation in which the injected fluid is now more viscous and more dense than the displaced fluid. Since in the absence of rotation this setup is obviously stable regarding viscosity difference, we focus on the case in which the cell rotates, so that the outgrowing diffusing interface is centrifugally unstable. Moreover, in order to produce significant fingering within the computational domain, we take a large value of $\Omega$. Coriolis force effects are also discussed. At the same time, to avoid possible problems with the resolution of the images and with the convergence properties of the numerical code, we consider a relatively lower value for the Péclet number. From Eq. (14) one can verify that for a fixed $\Omega$, a lower value of Pe means enhanced diffusion, which tends to stabilize interfacial disturbances. Figure 6 illustrates the resulting patterns obtained for $A$ $=0.874, \mathrm{Pe}=2.0 \times 10^{2}$, and $\Omega=10^{2}$ : on the top row Coriolis 
effects are neglected $\left(\operatorname{Re}_{1}=0, \operatorname{Re}_{2}=0\right)$, and on the bottom row they are included $\left(\operatorname{Re}_{1}=0.01, \mathrm{Re}_{2}=0.07\right)$. The first column in Fig. 6 depicts the patterns for $\delta=0$, and the second column shows the case in which $\delta=-10^{-4}$. In contrast to what we have done in previous figures of this section the snapshots of the plots shown in Fig. 6 are not taken at identical times. Instead, we terminate the simulations when the outermost finger reaches the location of ten grid points away from the computational boundaries. This is done to avoid boundary effects. Of course, depending on the physical parameters considered in each computational run, this specific location will be reached at different times. These values of time can be used to determine the degree of instability of the system, since shorter times indicate a more unstable situation.

The fact that in Fig. 6 the injected fluid is more viscous and of higher density result in patterns that are very different from those depicted in Figs. 2-4. In Fig. 6(a) we see the formation of interesting spiny structures containing long and thin, radially symmetric outward fingers, presenting a very uniform finger-length distribution. Note that the internal portion of the interface is fairy circular. The reason for this spiny structure is mainly due to the delayed action of the centrifugal force. It should be noted that the interface morphology is determined by the competition of stable outward displacement (injection) and unstable centrifugal driving. While the stabilizing role of injection is most significant at early time (when the interface is near the origin), the centrifugal force dominates at later times (when the interface far away from the origin). During the early times of stable displacement, the initial perturbations are smoothed out by diffusion. Later on, when the centrifugally-driven fingers start to develop, there are almost no preferences given by the initial perturbation, leading to an almost evenly distributed fingering pattern. In addition, we point out that the development of the nearly circular internal region is a good indication that no significant grid orientation effects occur in the present simulation.

The introduction of a nonzero Korteweg stress makes the interface more stable [Fig. 6(b)], leading to the formation of a smaller number of shorter and thicker fingers which are characterized by a significant finger length variability. All these morphological changes are reminiscent of the surfacetension-like role played by the Korteweg stresses. Similarly to what was observed in Fig. 6(a), the perimeter of the central droplet illustrated in Fig. 6(b) remains circular.

A curious effect is revealed in Figs. 6(c) and 6(d) where Coriolis effects are taken into account. Recall that in Fig. 4 (injection of the less viscous fluid) the most evident signature for the presence of the Coriolis force was the observation of bent fingers. However, in Figs. 6(c) and 6(d) instead of a significant finger bending, we observe a clear droplet body distortion, in which the internal region of the droplet is distorted from circularity, assuming a squarelike shape. A similar body distortion phenomenon is also revealed in threedimensional immiscible spin coating experiments [58] and numerical simulations [59]. It has also been found in previous simulations of miscible Hele-Shaw flow based on Schwartz's approach [33]. By contrasting Figs. 6(c) and 6(d) with Figs. 6(a) and 6(b) the stabilizing role of the Coriolis force is verified, so that the formation of long fingers is restrained.
We now turn to the discussion of the domination of body distortion over fingering formation and finger bending as illustrated in Fig. 6. When rotation is present, there are two major kinds of physical effects: (i) the rising of fingering due to centrifugal forces caused by increasing radial distance and radial velocity; (ii) the body distortion or finger bending due to the azimuthal Coriolis force. As demonstrated in a previous study of a purely rotating drop without injection [34], radial fingering occurs immediately. As time progresses, the length of the fingers keep increasing, resulting in large local velocities at the finger tips, while both the effective radius of drop's body and the local velocity nearby it are reduced. This leads to a very significant azimuthal force at the fingers' tips, so that finger bending is dominant. On the other hand, the presence of injection provides a very large local velocity to contribute to the azimuthal force at the drop's body, while fingering is restrained by the stabilizing viscous effects associated to the injection of the more viscous fluid. That explains why the body distortion is predominant earlier when injection is present. Nevertheless, finger bending will be also significant once the fingers reach a sufficiently large radial distance where strong local velocity is induced by a very long radius of gyration. This can be already seen by the slight angular deviation of the finger tips in Fig. 6(c), which are quite different from the straight and radially oriented fingering structures depicted in Fig. 6(a).

We conclude this section by examining Fig. 7 which depicts the streamlines (contour lines of the streamfunction) associated to the patterns shown in Fig. 6. The plot of the streamlines provides additional quantitative assessment of the miscible structures shown in Fig. 6, clearly illustrating the local velocity direction. Both parts of the streamfunction are included in Fig. 7 , the potential part $\phi_{\text {pot }}$ (associated to the source) and the rotational part $\phi$. It should be noted that the straight line appearing at the negative $x$ axis should be ignored. It appears as a result of the discontinuity of the streamfunction due to the source which is given by $\phi_{\text {pot }}=$ $-\pi\left(\phi_{p o t}=\pi\right)$ when it is approached in the counterclockwise (clockwise) direction.

For the cases in which the Coriolis force is absent [Figs. 7 (a) and 7(b)], basically no rotational velocity is generated in the inner region occupied by the more dense fluid, so that the streamlines grow radially outward due to the action of the source. For the same reason, in the region outside the mixing interface (which is mostly occupied by the lighter fluid), the potential radial streamlines are not significantly altered. Nevertheless, the concentration variation at the mixing region gives rise to a significant vorticity, and numerous eddy pairs are formed locally. Each one of these eddy pairs can be treated as local perturbing sources which originate a growing finger with a nearly axisymmetric distribution, as shown in the corresponding concentration images depicted in Figs. 6(a) and 6(b). Note that the number of eddy pairs is significant higher for the zero Korteweg stress case [Fig. 7(a)] leading to the formation of a larger number of fingers. Besides, the strength of the eddy pairs, which can be represented by the local density distribution of streamlines, is notably stronger in Fig. 7(a) than in Fig. 7(b). Therefore, the increased number as well as the large magnitude of the local eddy pairs confirm the enhanced instability associated to the case without Korteweg stresses. 
(a)

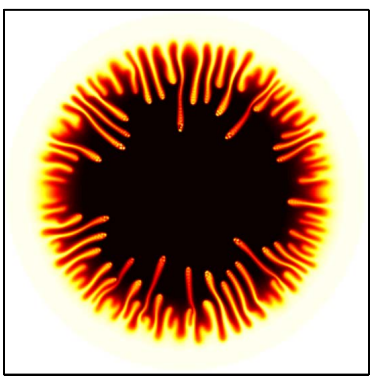

(b)

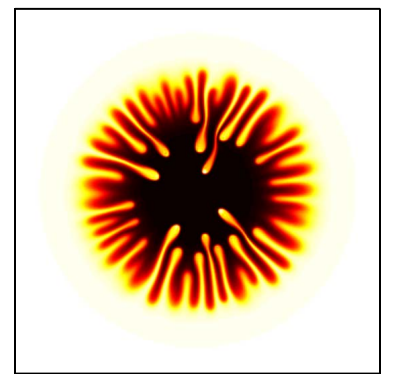

(c)

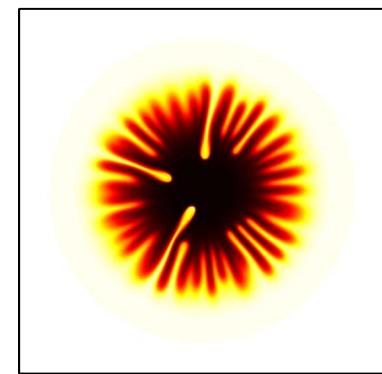

FIG. 8. (Color online) Concentration images for inward radial flow with $A=0.905, \mathrm{Pe}=2.0 \times 10^{3}, \Omega=0$, and $\operatorname{Re}_{1}=\mathrm{Re}_{2}=0$ for (a) $\delta=0$, $t=0.34$; (b) $\delta=-10^{-4}, t=0.41$; (c) $\delta=-2.0 \times 10^{-4}, t=0.43$.

If the Coriolis forces are taken into account [Figs. 7(c) and $7(\mathrm{~d})$ ], despite the essentially radial distribution of the streamlines in the inner region, and the formation of eddy pairs at the mixing region, now the streamlines clearly turn in the counterclockwise direction right outside the mixing interface. Of course, this is due to the action of the Coriolis force. Remember that since this is an outward radial flow, the streamlines are directed from inside to the outside region. Since the Coriolis forces acting against the actual rotating direction of the Hele-Shaw cell depend on fluid density as expressed in Eq. (2), the local azimuthal velocity would be smaller for a heavier inner fluid because of stronger Coriolis forces locally. This explains the rotating fingering orientation seen in Figs. 6(c) and 6(d).

\section{B. Inward radial flow}

As commented in Sec. I the shape of the patterns formed in the inward radial flow of immiscible fluids has been investigated by a couple of experiments $[3,4]$. It has been found that during the suction of the more viscous fluid, the initially circular interface shrinks and ramifies via the penetration of multiple fingers of the outer less viscous fluid into the inner one. Later on, a screening process takes place and eventually a single finger reaches the sink, while the remaining fingers basically stop growing. It is worth noting that, to date there are no equivalent Hele-Shaw studies (neither experimental nor numerical) addressing the miscible displacement for inward radial flow.

We examine the inward radial flow situation considering that the fluids involved are miscible. Since the overall size of the patterns decreases as the inner fluid is sucked out, we reduced the plots' frame size in order to facilitate the visualization of the morphological details of the structures at later times. Thus, all the patterns depicted in this section are plotted inside a square frame that has the dimensions given by $-0.6 \leqslant x \leqslant+0.6$ and $-0.6 \leqslant y \leqslant+0.6$. However, the dimensions of the computational domain remain unchanged, being $-4 / 3 \leqslant x \leqslant+4 / 3$ and $-4 / 3 \leqslant y \leqslant+4 / 3$. This set of numerical experiments illustrate patterns at a largest achievable time before our numerical code becomes unstable. As the inward moving fingers approach the sink, their local velocity becomes considerably high, producing significantly intense vorticity. So, to avoid numerical instabilities we take the final times as those for which the local vorticity reaches a certain maximum magnitude (taken as $\zeta_{\max }=2000$ ). For larger values of the vorticity $\left(\zeta>\zeta_{\max }\right)$ fingering is too vigorous and the code becomes unstable.

We have performed a number of numerical tests, and have explicitly verified that the suction of a less viscous fluid is indeed a stable pattern formation process both in the absence and presence of rotation, resulting in a contracting circular interface. Thus, we focus on the unstable situation regarding suction, and examine the case in which the inner fluid is more viscous and of higher density than the outer fluid. First, we investigate the role of Korteweg stresses in the case that the cell is not rotating so that only suction is acting. Figure 8 plots the resulting miscible patterns when the more viscous and more dense fluid is withdrawn from the system for Pe $=2.0 \times 10^{3}, A=0.905$ with (a) $\delta=0$, at $t=0.34$; (b) $\delta=-10^{-4}$, at $t=0.41$; (c) $\delta=-2.0 \times 10^{-4}$, at $t=0.43$. When $\delta=0$ the typical miscible patterns are quite different from those obtained experimentally for the suction of immiscible fluids [3,4]. In Fig. 8(a) we observe the formation of a highly fragmented pattern, in which a very large number of thin invading fingers of the less viscous fluid compete as they move toward the sink. A nonzero Korteweg stress is considered in Fig. 8(b) leading to a less convoluted structure that presents a smaller number of invading fingers. These fingers are typically thicker than the ones shown in Fig. 8(a), and their ends are more bulbous. The stabilizing role of the Korteweg stresses is evidenced by the fact that the maximum time taken in Fig. 8(b) is larger than the one obtained in Fig. 8(a).

The magnitude of $\delta$ is further increased in Fig. 8(c) resulting in a pattern for which only a couple of fingers actually can "win the race" toward the center. The remaining fingers which have been left behind are screened off, and their velocities are significantly reduced. Although the general properties of the pattern illustrated in Fig. 8(c) for the highest value of $\delta$ are in line with the ones experimentally detected for inward radial flows with immiscible fluids [4], the agreement between their specific morphological features is not that good. This is in part due to the fact that the immiscible experiments in Ref. [4] take $A=1$, while the miscible simulation shown in Fig. 8(c) considers that $A=0.905$ [with $\delta=-2.0 \times 10^{-4}$ ]. However, a much better agreement between our numerical simulations and the typical experimental pattern found in [4] is obtained in Fig 9(c), where we have used a higher value for the viscosity contrast $(A$ $=0.92$ ), a larger magnitude for the highest Korteweg stress 
(a)

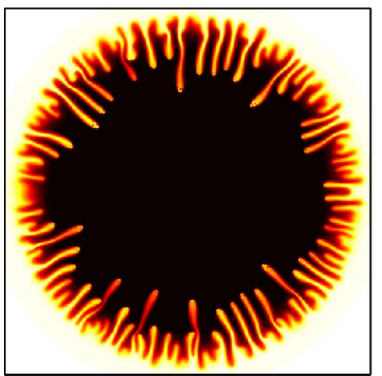

(b)

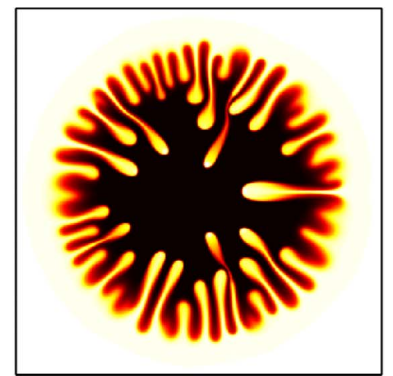

(c)

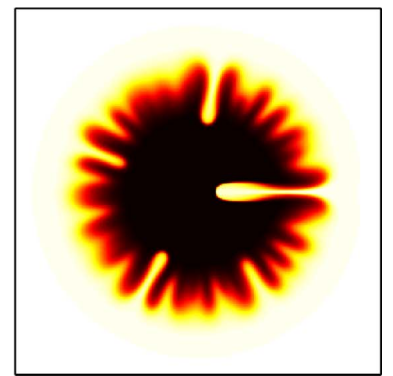

FIG. 9. (Color online) Concentration images for inward radial flow with $A=0.92, \mathrm{Pe}=2.0 \times 10^{3}, \Omega=0$, and $\operatorname{Re}_{1}=\mathrm{Re}_{2}=0$ for (a) $\delta=0, t$ $=0.26$; (b) $\delta=-10^{-4}, t=0.36$; (c) $\delta=-5.0 \times 10^{-4}, t=0.39$.

parameter $\left(\delta=-5.0 \times 10^{-4}\right)$, in conjunction with a slight modification of the initial conditions with respect to the ones used in Fig. 8. As illustrated in Fig 9(c) the increase in $\delta$ favors the penetration of a smaller number of fingers, and eventually only a longer dominant finger reaches the center. For completeness, Figs. 9(a) and 9(b) are plotted for lower values of $\delta$. Even though the patterns shown in Figs. 9(a) and 9(b) are somewhat similar to the corresponding structures illustrated in Figs. 8(a) and 8(b), Figs. 9(c) and 8(c) are markedly different. Most importantly, the miscible pattern simulated in Fig. 9(c) for larger $\delta$ has a much closer resemblance with the experimental immiscible pattern depicted in Fig. 15 of Ref. [4]. It is in fact reassuring to see that the immiscible behavior for inward radial flow is correctly recovered by our numerical simulations when $\delta$ is sufficiently large.

The role of rotation for inward radial flow is analyzed in Fig. 10. Unfortunately, since rotation destabilizes the system even further, numerical instabilities arise so that we could not simulate for $A=0.92$ as we did in Fig. 9, but instead for $A=0.905$ as in Fig. 8. So, Fig. 10 considers the same physical parameters and initial condition employed in Fig. 8, but now adding rotational effects. More precisely, Fig. 10 illustrates the resulting patterns obtained for $A=0.905, \mathrm{Pe}=2.0$ $\times 10^{3}$, and $\Omega=3$ : on the top row Coriolis effects are neglected $\left(\operatorname{Re}_{1}=0, \operatorname{Re}_{2}=0\right)$, and on the bottom row they are included $\left(\operatorname{Re}_{1}=0.01, \mathrm{Re}_{2}=0.06\right)$. Moreover, the first (second) column considers that $\delta=0\left(\delta=-10^{-4}\right)$. To investigate the role of rotation, we begin by contrasting Figs. 10(a) and 10(b) to Figs. 8(a) and 8(b), respectively. By inspecting these figures it is clear that the simple addition of centrifugal forces does not produce dramatic changes in the shape of the patterns. Of course, rotation drives the system more unstable and makes the invading fingers to approach the sink more rapidly. In addition, the inward fingers in Fig. 10(b) look a little thicker and present more inflated tips than in Fig. 8(b).

A completely different scenario is observed when the Coriolis effects are taken into consideration [Figs. 10(c) and 10(d)]. By comparing Fig. 10(a) to Fig. 10(c), and Fig. 10(b) to Fig. 10(d), we see that the morphology of the patterns are drastically modified: the number of inward fingers is considerably reduced, and their trajectories are clearly deviated from the radial direction. During sucking the inward fingers meander toward the sink and tend to bend in the clockwise direction, while the more external parts of the outer fluid form "petals" which turn in the counterclockwise direction. The result are patterns [in particular the one depicted in Fig. $10(\mathrm{~d})]$ that resemble four-leaf clovers. As expected, both pattern stabilization and finger bending are typical effects caused by the Coriolis force. However, the precise morphological changes introduced by the Coriolis effects are decidedly nontrivial, and a little hard to be predicted without resorting to full numerical simulations of the advanced nonlinear stages.

The streamlines corresponding to the patterns shown in Fig. 10 are illustrated in Fig. 11 (as in Fig. 7 please neglect the straight line located along the negative $x$ axis). When Coriolis effects are neglected [Figs. 11(a) and 11(b)] we observe the clear dominance of radially oriented streamlines (due to the influence of the sink), which are only modestly altered by a few eddy pairs at the regions where fingering is active. This is very different from behavior of the injection cases analyzed in Figs. 7(a) and 7(b), where we had a mostly radially oriented distribution of streamlines interrupted by a significant larger number of eddy pairs located at the mixing interface. The reason for the formation of a smaller number of eddy pairs in Figs. 11(a) and 11(b) can be attributed to the prevalence of the potential sink flow over the rotating flow components in the inward flow case. The rotational component is perturbed by the vorticity produced by viscous effects associated with the local velocity. In contrast, the potential component is related to the sink flow that depends inversely on the radial distance. Since in Figs. 11(a) and 11(b) fluids travel toward to the origin, the major contribution for these streamlines will be due to the potential inward flow. In addition, the formation of a few more eddy pairs in Fig. 11(b) can be explained by the fact that in this case fingers can move closer to the origin, where higher local potential velocity would generate a more significant vorticity, so that the rotational component velocity would be more intense, resulting in the formation of some eddy pairs.

Figures 11(c) and 11(d) display the streamlines if the Coriolis forces are taken into consideration. For the same reasons presented in the analysis of the streamlines for the injection case (Fig. 7), more intense Coriolis forces opposing counterclockwise fluid rotation are imposed to the more dense inner fluid. As a result, similar types of streamline patterns of those shown in Figs. 7(c) and 7(d) are obtained in Figs. 11(c) and 11(d). However, recall that in the inward flow case of Fig. 11 the streamlines travel toward the sink (they 
(a)

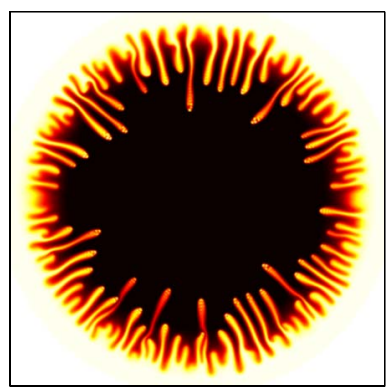

(c)

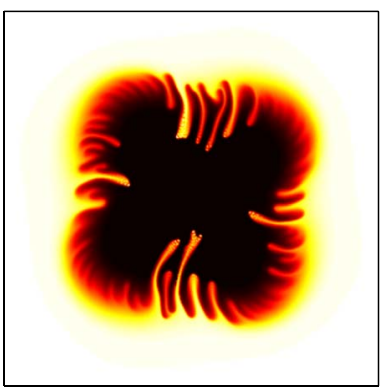

(d)

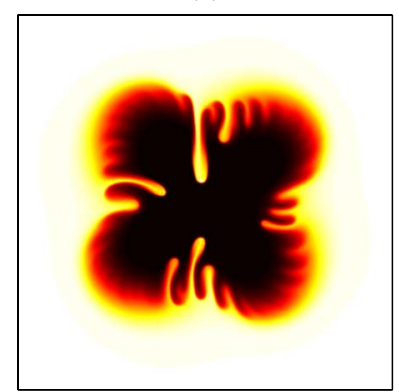

FIG. 10. (Color online) Concentration images for $A=0.905$, Pe $=2.0 \times 10^{3}$, and $\Omega=3$, when $[(\mathrm{a})$ and (b)] Coriolis effects are neglected $\left(\operatorname{Re}_{1}=0, \operatorname{Re}_{2}=0\right)$ for (a) $\delta=0, t=0.29$, (b) $\delta=-10^{-4}, t$ $=0.38$; and when $[(\mathrm{c})$ and (d)] the Coriolis force is taken into account $\left(\mathrm{Re}_{1}=0.01, \mathrm{Re}_{2}=0.06\right)$ considering that (c) $\delta=0, t=0.37$; (d) $\delta=-10^{-4}, t=0.39$.

are directed from the outside to the inside region), meaning that they actually turn in the clockwise direction. This explains the clockwise deviation of the invading (inward moving) fingers as shown in the concentration images in Figs. $10(\mathrm{c})$ and $10(\mathrm{~d})$.

\section{CONCLUDING REMARKS}

In this work, we have revisited the immiscible radial fingering problem in Hele-Shaw cells, now considering that the fluids are miscible. In addition to injection or suction, the confined system can be subjected to rotation, so that centrifugal and Coriolis forces may affect the evolution of the diffusing interface in a nontrivial manner. This complex pattern formation problem is examined in detail through intensive numerical simulations.

Our numerical experiments reveal that, when fingering is initiated between miscible fluids, the lack of surface tension at the moving interface results in patterns which are much richer in detail and gross structure. In fact, we have observed dramatic changes in pattern morphology as the main control parameters of the problem are varied. Depending on the balance among injection, suction, diffusive, and rotational effects a gallery of pattern-forming structures is obtained, ranging through fragmented, $\operatorname{cog}$ wheel, pinwheel shaped, spiny, and cloverlike patterns. In addition to unveiling a wide variety of shapes, we have verified that the miscible patterns can be made to resemble the more familiar morphologies of their immiscible counterparts (i.e., typical fanlike patterns (a)

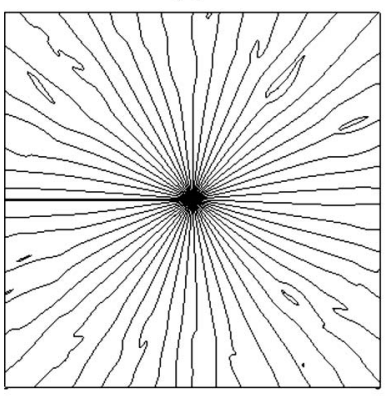

(c)

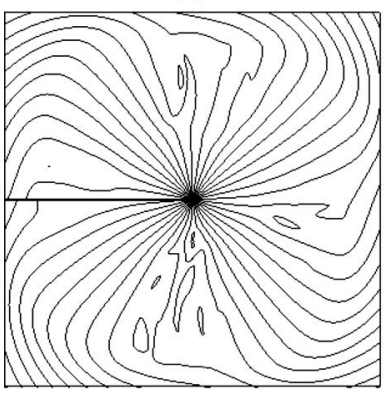

(b)

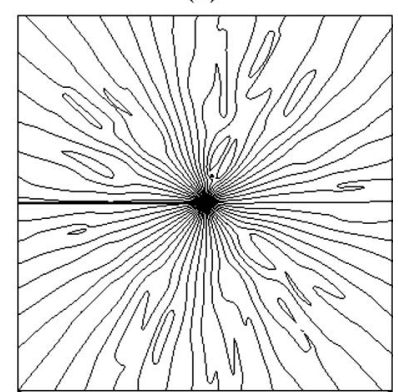

(d)

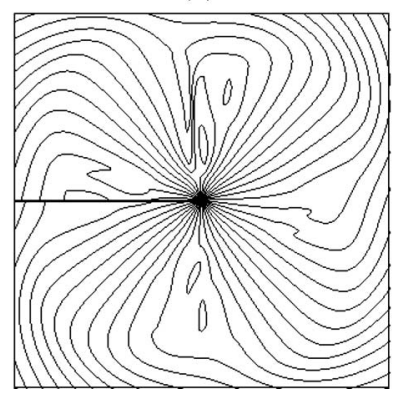

FIG. 11. Streamlines of the situations shown in Fig. 10

for outward flow, and a characteristic hierarchy of screenedoff fingers for inward flow) by conveniently increasing the value of a Korteweg stress parameter. This finding is certainly reassuring, since it substantiates the surface tensionlike role of such stresses for miscible flows, and also indicates the effectiveness and reliability of our numerical scheme.

When contrasted to existing laboratory experiments and other theoretical or numerical studies, the general features of the simulated miscible fronts usually reveal good agreements. We point out that some of the predicted shapes obtained as the result of our simulations have not yet been checked by experiments. Needless to say that we welcome and encourage experimentalists to perform such studies, and test our numerical predictions. On the other hand, we also note that a few detailed structural features revealed by existing experiments with miscible fluids $[10,11]$ have not been completely captured by our numerical simulations. For example, we have not reproduced the "flowerlike" patterns obtained by Paterson [10], and the peculiar sidebranched structures found by Chen [11].

Although the specific reasons that could justify our incapacity to reproduce a few features of some specific miscible patterns are not at all clear, on the theoretical side one could speculate that this maybe linked to the limitations of the Hele-Shaw approximation [28], indicating that under certain circumstances the concentration gradients can indeed become high across the gap. If this is the case, threedimensional effects $[44-46,60]$ may have a significant role in determining the shape of the emerging patterns, so that full 3D Stokes equations or the Brinkman model could be more appropriate to capture the instability features. Unfortunately, the numerical implementation of these two schemes are still considerably difficult. Nevertheless, there is also the possibility that some of the detailed features found in these 
experiments $[10,11]$ may come from physical ingredients still not considered by our theoretical model (and for that matter, maybe also overlooked in $[10,11]$ ), such as the possible influence of non-Newtonian aspects (viscoelasticity $[61,62]$, shear-thinning, and shear-thickening effects [63]) on certain miscible displacements. Another possibly relevant aspect for radial miscible fingering, recently investigated by Nagatsu et al. [64] involves viscosity changes due to variation in chemical species concentrations caused by chemical reactions. Of course, the search for more quantitative relations between the observed miscible patterns and the physical properties of the fluids is a very exciting, challenging, and still open topic that deserves attention from both theorists and experimentalists.

\section{ACKNOWLEDGMENTS}

J.A.M. acknowledges CNPq (Brazilian Research Council) for financial support of this research through the program "Instituto do Milênio de Fluidos Complexos" Contract No. 420082/2005-0, and also through the CNPq/FAPESQ Pronex program. H.G. acknowledges financial support from FACEPE (Fundação de Amparo à Ciência e Tecnologia do Estado de Pernambuco, Brazil) through the Contract BPD No. 0008-1.05/07. C.-Y.C. acknowledges the National Science Council of the Republic of China for financial support of this research through Grant No. NSC 95-2221-E224-052.
[1] For review articles see for instance, D. Bensimon, L. P. Kadanoff, S. Liang, B. I. Shraiman, and C. Tang, Rev. Mod. Phys. 58, 977 (1986); G. Homsy, Annu. Rev. Fluid Mech. 19, 271 (1987); K. V. McCloud and J. V. Maher, Phys. Rep. 260, 139 (1995); J. Casademunt, Chaos 14, 809 (2004).

[2] P. G. Saffman and G. I. Taylor, Proc. R. Soc. London, Ser. A 245, 312 (1958).

[3] L. Paterson, J. Fluid Mech. 113, 513 (1981).

[4] H. Thomé, M. Rabaud, V. Hakim, and Y. Couder, Phys. Fluids A 1, 224 (1989).

[5] O. Praud and H. L. Swinney, Phys. Rev. E 72, 011406 (2005).

[6] P. Fast and M. J. Shelley, J. Comput. Phys. 212, 1 (2006).

[7] J. Mathiesen, I. Procaccia, H. L. Swinney, and M. Thrasher, Europhys. Lett. 76, 257 (2006).

[8] H. D. Ceniceros, T. Y. Hou, and H. Si, Phys. Fluids 11, 2471 (1999).

[9] H. D. Ceniceros and H. Si, J. Comput. Phys. 165, 237 (2000).

[10] L. Paterson, Phys. Fluids 28, 26 (1985).

[11] J.-D. Chen, J. Fluid Mech. 201, 223 (1989); Exp. Fluids 5, 363 (1987).

[12] M.-N. Pons, E. M. Weisser, H. Vivier, and D. V. Boger, Exp. Fluids 26, 153 (1999).

[13] C. T. Tan and G. M. Homsy, Phys. Fluids 30, 1239 (1987).

[14] Y. C. Yortsos, Phys. Fluids 30, 2928 (1987).

[15] D. Joseph, Eur. J. Mech. B/Fluids 9, 565 (1990).

[16] D. Korteweg, Arch. Neerl. Sci. Exactes Nat., Ser. II 6, 1 (1901).

[17] H. Hu and D. Joseph, ZAMP 43, 626 (1992).

[18] C.-Y. Chen and E. Meiburg, Phys. Fluids 14, 2052 (2002).

[19] J. A. Pojman, C. Whitmore, M. L. T. Liveri, R. Lombardo, J. Marszalek, R. Parker, and B. Zoltowski, Langmuir 22, 2569 (2006).

[20] B. Zoltowski, Y. Chekanov, J. Masere, J. A. Pojman, and V. Volpert, Langmuir 23, 5522 (2007).

[21] L. W. Schwartz, Phys. Fluids A 1, 167 (1989).

[22] Ll. Carrillo, F. X. Magdaleno, J. Casademunt, and J. Ortín, Phys. Rev. E 54, 6260 (1996).

[23] E. Alvarez-Lacalle, J. Ortín, and J. Casademunt, Phys. Fluids 16, 908 (2004).

[24] F. X. Magdaleno, A. Rocco, and J. Casademunt, Phys. Rev. E 62, R5887 (2000).
[25] E. Alvarez-Lacalle, E. Pauné, J. Casademunt, and J. Ortín, Phys. Rev. E 68, 026308 (2003).

[26] J. A. Miranda and E. Alvarez-Lacalle, Phys. Rev. E 72, 026306 (2005).

[27] C.-Y. Chen and S.-W. Wang, Fluid Dyn. Res. 30, 315 (2002).

[28] C.-Y. Chen, C.-H. Chen, and J. A. Miranda, Phys. Rev. E 73, 046306 (2006).

[29] S. L. Waters and L. J. Cummings, Phys. Fluids 17, 048101 (2005).

[30] S. L. Waters, L. J. Cummings, K. M. Shakesheff, and F. R. A. J. Rose, IMA J. Math. Appl. Med. Biol. 23, 311 (2006).

[31] H. Gadêlha, N. Brito, and J. A. Miranda, Phys. Rev. E 75, 016305 (2007).

[32] A. Abidate, S. Aniss, O. Caballina, and M. Souhar, Phys. Rev. E 75, 046307 (2007).

[33] C.-Y. Chen and Y.-C. Liu, Int. J. Numer. Methods Fluids 48, 853 (2005).

[34] C.-H. Chen and C.-Y. Chen, Int. J. Numer. Methods Fluids 51, 881 (2006); 54, 1201 (2007).

[35] C.-Y. Chen, C.-H. Chen, and J. A. Miranda, Phys. Rev. E 71, 056304 (2005).

[36] C. T. Tan and G. M. Homsy, Phys. Fluids 31, 1330 (1988).

[37] C.-Y. Chen and E. Meiburg, J. Fluid Mech. 371, 233 (1998); 371, 269 (1998).

[38] P. Petitjeans and T. Maxworthy, J. Fluid Mech. 326, 37 (1996).

[39] C.-Y. Chen and E. Meiburg, J. Fluid Mech. 326, 57 (1996).

[40] G. I. Taylor, Proc. R. Soc. London, Ser. A 219, 186 (1953).

[41] L. J. Cummings and S. L. Waters, IMA J. Math. Appl. Med. Biol. 24, 169 (2007).

[42] E. Lajeunesse, J. Martin, N. Rakotomalala, and D. Salin, Phys. Rev. Lett. 79, 5254 (1997).

[43] E. Lajeunesse, J. Martin, N. Rakotomalala, D. Salin, and Y. Yortsos, J. Fluid Mech. 398, 299 (1999).

[44] J. Fernandez, P. Kurowski, L. Limat, and P. Petitjeans, Phys. Fluids 13, 3120 (2001)

[45] J. Fernandez, P. Kurowski, P. Petitjeans, and E. Meiburg, J. Fluid Mech. 451, 239 (2002).

[46] F. Graf, E. Meiburg, and C. Hartel, J. Fluid Mech. 451, 261 (2002).

[47] A. Riaz and E. Meiburg, Phys. Fluids 15, 938 (2003). 
[48] A. Riaz, C. Pankiewitz, and E. Meiburg, Phys. Fluids 16, 3592 (2004).

[49] G. Tryggvason and H. Aref, J. Fluid Mech. 136, 1 (1983).

[50] E. Meiburg and C.-Y. Chen, SPEJ 5, 129 (2000).

[51] D. Gottlieb and S. A. Orzag, Numerical Analysis of Spectral Methods (SIAM, Philadelphia, 1977).

[52] C. Canuto, M. Y. Hussaini, A. Quarteroni, and T. A. Zang, Spectral Methods in Fluid Mechanics (Springer, Berlin, 1988).

[53] S. K. Lele, J. Comput. Phys. 103, 16 (1992).

[54] M. Ruith and E. Meiburg, J. Fluid Mech. 420, 225 (2000).

[55] A. Wray (unpublished).

[56] C.-Y. Chen, L. L. Wang, and E. Meiburg, Phys. Fluids 13,
2447 (2001).

[57] C.-Y. Chen, Phys. Fluids 15, 1086 (2003).

[58] N. Fraysse and G. M. Homsy, Phys. Fluids 6, 1491 (1994).

[59] L. W. Schwartz and R. V. Roy, Phys. Fluids 16, 569 (2004).

[60] N. Goyal and E. Meiburg, J. Fluid Mech. 516, 211 (2004).

[61] H. Zhao and J. V. Maher, Phys. Rev. A 45, R8328 (1992).

[62] T. Podgorski, M. C. Sostarecz, S. Zorman, and A. Belmonte, Phys. Rev. E 76, 016202 (2007).

[63] L. Kondic, M. J. Shelley, and P. Palffy-Muhoray, Phys. Rev. Lett. 80, 1433 (1998).

[64] Y. Nagatsu, K. Matsuda, Y. Kato, and Y. Tada, J. Fluid Mech. 571, 475 (2007). 